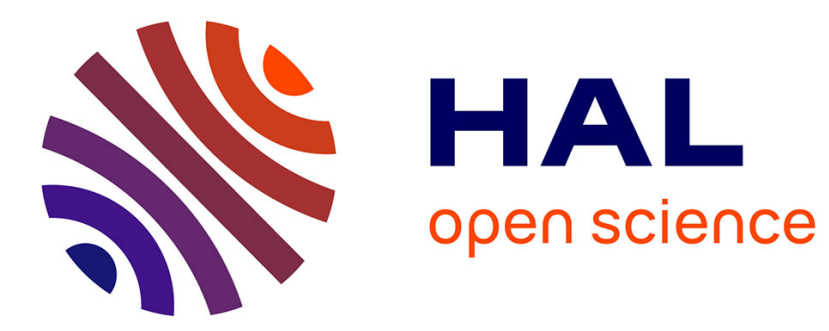

\title{
An Interactive EA for Multifractal Bayesian Denoising
}

Evelyne Lutton, Pierre Grenier, Jacques Lévy Véhel

\section{To cite this version:}

Evelyne Lutton, Pierre Grenier, Jacques Lévy Véhel. An Interactive EA for Multifractal Bayesian Denoising. EvoIASP 2005, Mar 2005, Lausanne, Suisse. pp.1. hal-00539290

\section{HAL Id: hal-00539290 \\ https://hal.science/hal-00539290}

Submitted on 24 Jan 2011

HAL is a multi-disciplinary open access archive for the deposit and dissemination of scientific research documents, whether they are published or not. The documents may come from teaching and research institutions in France or abroad, or from public or private research centers.
L'archive ouverte pluridisciplinaire HAL, est destinée au dépôt et à la diffusion de documents scientifiques de niveau recherche, publiés ou non, émanant des établissements d'enseignement et de recherche français ou étrangers, des laboratoires publics ou privés. 


\title{
An Interactive EA for Multifractal Bayesian Denoising
}

\author{
Evelyne LUTTON, Pierre GRENIER and Jacques LEVY VEHEL \\ INRIA - COMPLEX Team, \\ B.P. 105, 78153 Le Chenay cedex, France \\ http://fractales.inria.fr
}

\begin{abstract}
We present in this paper a multifractal bayesian denoising technique based on an interactive EA. The multifractal denoising algorithm that serves as a basis for this technique is adapted to complex images and signals, and depends on a set of parameters. As the tuning of these parameters is a difficult task, highly dependent on psychovisual and subjective factors, we propose to use an interactive EA to drive this process. Comparative denoising results are presented with automatic and interactive EA optimisation. The proposed technique yield efficient denoising in many cases, comparable to classical denoising techniques. The versatility of the interactive implementation is however a major advantage to handle difficult images like IR or medical images.
\end{abstract}

\section{Introduction}

Interactive Evolutionary Algorithms (IEA) have now many applications in various domains, where quantities to be optimised are related to subjective rating (visual or auditive interpretation). Following founding works, $[12,11,15,1]$ oriented towards artistic applications, characteristic examples are [14] for Hearing Aids fitting, [5] for smooth, human-like, control rules design for a robot arm, or [9] for the design of HTML style sheets. An overview of this vast topic can be found in [13].

The specific context of human interaction constrains the evolutionary engine a different way as classical EA approaches. The "user bottleneck" [10], i.e. the human fatigue, is a major fact. Solutions have to be found in order to efficiently drive the evolution of the system while avoiding systematic and boring interactions [10,13,2]. Usually, the IEA populations are small, and interfaces are designed in order to let the user interact in various ways with evolution (initialisations, solutions rating, and if possible direct modifications on genomes [3]).

The present work deals with complex image analysis techniques that depend from a set of control parameters. These techniques are precise and efficient, but depending on applications, the aim of the end-used may be very different (medical practicionner, teledetection, stereophotogrametry), and some judgment criteria are fully user dependent.

We explore the idea of developing an interactive EA to cope with unpredictibility of the exact aim of the user. As an example, for denoising applications, computed image distance is not sufficient to decide which algorithm is the best. Criteria related to 
details preservation, and depending on psychovisual factors are extremely important. Moreover, the end-user judgment depends on the way he will use the denoised image ...

The paper is organised as follows. In section 2 the principle of the multifractal bayesian denoising technique is presented, and the free parameters are identified. These free parameters are optimised with the help of an interactive evolutionary algorithm, see section 3. Results of interactive and automatic optimisations are presented in section 4, with comparisons with another efficient denoising technique based on wavelet coefficients. Conclusions and future work are presented in section 5.

\section{Multifractal bayesian denoising}

\subsection{Multifractal analysis}

The multifractal analysis of a signal consists in measuring its regularity at each sample point, in grouping the points having the same irregularity, and then in estimating the Hausdorff dimension (i.e. the "fractal dimension") of each iso-regularity set. Irregularity is measured via the local Hölder exponent [7] defined for a continuous function $f$ at $x_{0}$ as the largest real $\alpha$ such that:

$$
\exists C, \rho_{0}>0: \forall \rho<\rho_{0} \quad \sup _{x, y \in B\left(x_{0}, \rho\right)} \frac{|f(x)-f(y)|}{|x-y|^{\alpha}} \leq C
$$

Since $\alpha$ is defined at each point, we may associate to $f$ the function $x \rightarrow \alpha(x)$ which measures the evolution of its regularity.

A multifractal spectrum $f_{H}$ is a representation of the irregularity of the signal over its definition domain. For each irregularity value, i.e. for each $\alpha$, one estimates $f_{H}(\alpha)$ as the Hausdorff dimension (the "fractal dimension," intuitively related to a frequency/geometrical distribution) of the corresponding iso- $\alpha$ set. As an example, for image data, a $f_{H}(\alpha) \simeq 1$ corresponds to a linear and smooth structure, while $f(\alpha) \simeq 0$ is a set of scattered points (singular points), or $f(\alpha) \simeq 2$ is a uniformly textured area.

The multifractal spectrum is a central notion exploited in multifractal image and signal analysis, as it provides in the same time a local $(\alpha)$ and a global $\left(f_{H}(\alpha)\right)$ viewpoint on data. It has been exploited with success in many applications where irregularity bears some important informations (image segmentation [6], signal and image denoising [7], etc ... )

Wavelet transforms are convenient tools for the estimation of the Hölder exponents. Our method is thus based on a discrete wavelet transform, and has been compared to another denoising technique based on wavelets (soft thresholding), know as very efficient in many cases, see section 4.

\subsection{Bayesian denoising}

The principle of the method is the following: For a noisy image $I_{1}$, we search for a denoised image $I_{2}$ that satisfies two conditions:

- $I_{2}$ has a given multifractal spectrum, 
- the probability that the addition of a white gaussian noise (with variance $\sigma$ ) to $I_{2}$ produces an observed image $I_{1}$, is maximal.

If the wavelet coefficient of the noisy image at scale $j$ is $y$, we get the following coefficient at the same scale for the denoised image (for details, see [8]):

$$
\widehat{x}=\operatorname{argmax}_{x>0}\left(j g\left(\frac{\log _{2}(\widehat{K} x)}{-j}\right)-\frac{(|y|-x)^{2}}{2 \sigma^{2}}\right) \operatorname{sgn}(y)
$$

where

- $\widehat{K}$ is a constant (that can depend from the scale $j$ ) such that $\widehat{K}|y|<1$ for every coeficients $y$ at scale $j$ of the noisy image. In what follows, $\widehat{K}$ is taken as the inverse of the maximal coefficient of each scale.

- $g$ is the function that defines the a priori spectrum of the denoised image.

We have chosen to use functions that verify the following properties:

- $g$ is defined on a interval $\left[\alpha_{\min }, \alpha_{\max }\right]$,

- $g(x) \in[0,1]$,

- there exists an $\alpha_{\text {mod }} \in\left[\alpha_{\text {min }}, \alpha_{\text {max }}\right]$ such that $g\left(\alpha_{\text {mod }}\right)=1$,

- $g$ is affine on $\left[\alpha_{\text {min }} ; \alpha_{m o d}\right]$ and on $\left[\alpha_{\text {mod }} ; \alpha_{\text {max }}\right]$.

The $g$ functions are thus fully determined by 5 values: $\alpha_{\min }, \alpha_{\bmod }, \alpha_{\max }, g\left(\alpha_{\min }\right)$ and $g\left(\alpha_{\max }\right)$.

As far as the previous set of values is chosen, the computation of the optimal wavelet coefficients of equation (1) is a simple deterministic calculation (as the a priori spectrum $g$ is affine by parts). The results provided by the method are fully determined.

\subsection{Free parameters}

A full set of parameters needs to be tuned in order to produce an efficient denoising with the previous method. Among them ${ }^{1}$, the most important ones are the values $\alpha_{\text {min }}$, $\alpha_{\bmod }, \alpha_{\max }, g\left(\alpha_{\min }\right)$ and $g\left(\alpha_{\max }\right)$, that define the profile of the a priori spectrum. For image denoising, these values have to be defined for the horizontal/vertical coefficients and for the diagonal ones.

Actually, we have chosen to distinguish the a priori hypotheses made on the horizontal/vertical coefficients and on the diagonal ones. Usually, on "non-noisy" images, the diagonal wavelet coefficients are significantly lower than the horizontal and vertical ones, while if these images are perturbed by an additive gaussian noise, this discrepancy

\footnotetext{
${ }^{1}$ The choice of the wavelet basis is of course important, and visually influences the results. For the present work we have chosen to optimise this parameter offline, independently from the interactive evolution process. Inded, experiments have been performed whith a genome including a symbolic component identifying the wavelet basis. It has been noticed that experimentally the wavelet coefficients that yield best results where Daubechies 8 to 12. It has however been noticed that as far as a "correct" wavelet has been chosen, the shape of the a priori spectrum is determining for the quality of results.
} 
vanishes $^{2}$. Diagonal wavelet coefficients seems to be more sensitive to additive noise, and a "stronger" denoising of them often yield better results.

We have thus chosen to use a different $g$ function for the diagonal coefficients: $g_{\text {Diag }}$ is similar to $g$, but translated with respect to its abcissa. This translation is an additional parameter of the method.

\section{Interactive optimisation of free parameters}

In [7], the multifractal denoising technique was based on another hypothesis with respect to the multifractal spectrum. We supposed that the noise was resulting into a translation of the multifractal spectrum of the initial image. In this paper, we relax this hypothesis and do not suppose that the spectrum of the initial image can be deduced from the degraded one. Moreover we do not suppose we know the variance of the noise (that we still suppose to be a white Gaussian noise however).

The resulting method is thus more versatile, but in the same time the quality of results is stongly dependent from the choice of the parameter set. The solution we propose is to let a human user choose among the various parameters combinations. This problem is of course strongly combinatorial and an interactive evolutionary algorithm has been designed in order to focus the search.

The population is made of a small number of individuals. Each individual is a parameter setting. It is presented to the user as an image, result of the corresponding denoising algorithm. The initial image (noisy) is simulatneously presented in the interface, so that the user can easily compare the results, see figure 1 .

\subsection{Genome}

The genome that will be evolved by the IEA is made of 7 real genes:

- 5 values to define the $g$ function used in formula (1) for the horizontal/vertical wavelet coefficients: ranges are chosen in order to ensure that the general shape of the spectrum is respected, i.e. $\alpha_{\text {min }} \in[0,0.5], g\left(\alpha_{\min }\right) \in[0,0.1], \alpha_{\bmod }>\alpha_{\min }$, $\alpha_{\bmod } \in[0,1], \alpha_{\max }>\alpha_{\bmod }, \alpha_{\max } \in[0.5,5], g\left(\alpha_{\max }\right) \in[0.9,1]$

- the shift of the $g$ function for the diagonal coefficients (range $[0,0.5]$ ),

- the noise variance $\sigma$ (range $[3.0,40.0]$ ).

\subsection{Fitness and user interaction}

The fitness function is given by the user via a cursor attached to each denoising result of the window. The range of values is $[-10,10]$. A default value of " 0 " (indifferent)

\footnotetext{
${ }^{2}$ Diagonal coefficients are roughly related to a second derivative of the signal while the horizontal/vertical ones are related to a first derivative. Additionally, this behaviour has been verified in a simple experiment on a set of 80 sample images: the mean values of horizontal/vertical wavelet coefficients have been computed and compared to the mean value of the diagonal ones. In average the quotient (mean diagonal values) over (mean vertical/horizontal values) is 0.52 (standard deviation 0.16).
} 


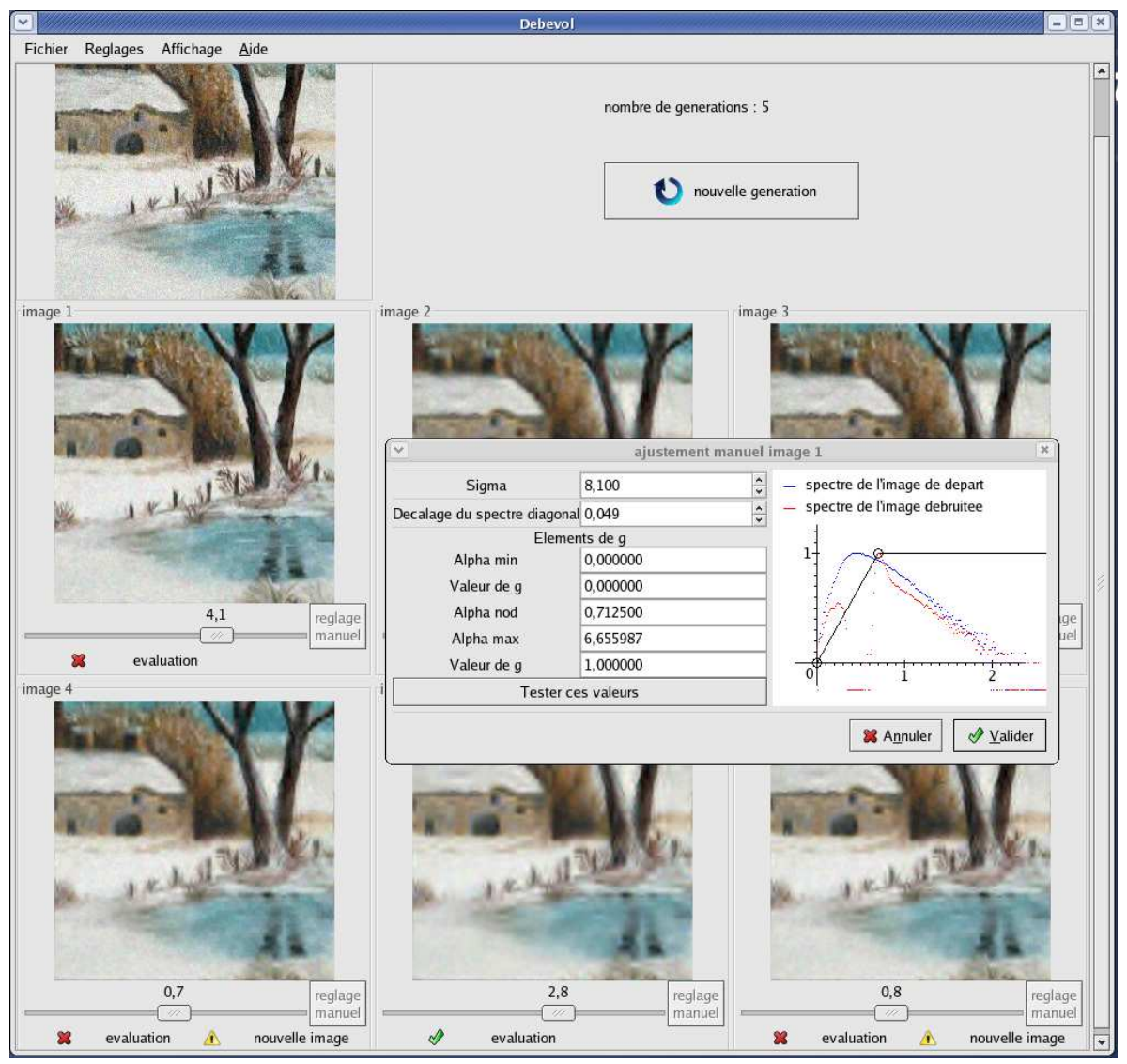

Fig. 1. Interface of the IEA

is set for each new genome (each image in the interface), the user can either increase, decrease or accept this value.

A sharing function is used in the selection process, in order to maintain diversity in the small population. The sharing is based on a weigthed $L_{2}$ distance computed on the real genes (parameters).

\subsection{Genetic engine}

The replacement step of the algorithms consists in replacing the 3 worst individuals of the population by new ones.

- Selection is performed by tournament of size 3 .

- Crossover is a barycentric crossover (the new individual is a weighted combination of his parents with a randomly chosen weight in $[0,1])$. 
- Mutation is an independent uniform perturbation of each gene value within a given range.

\subsection{Interaction interface}

When a new image to be denoised is loaded in the interface, 6 images are displayed that correspond to 6 initial random genomes with values within the range of admissible values. The user interacts with the system either by affecting a notation to some images of the current popUlation (cursor : 10 is good, -10 is bad), or by directly modifying the values of the parameter via a specialised window that appear when clicking on "manual interaction": results are directly observable inside the window and on the associated spectra. The result can be included in the current population and evolve the same way as automatically generated individuals.

This direct interaction is thus to be considered as an additionnal genetic operator, fully driven by user interaction (this is a factor that reduces the "user fatigue", by letting him the possibility to be more or less directive in the evolution process). The production of a new generation is triggered by a "next generation" button. Experimentally, this direct interaction appears as a important component for the efficiency of the evolution, and it allows the user to gain intuition -to some extent- on the influence of some of the genome parameters.

The whole set of EA parameters (genome values ranges, probabilities and various parameters associated to the genetic operators) are tunable via a specialised window. The image display can also be tuned for large images, in order to be able to have a global view on the whole population with reduced resolution, and a precise view to look at the details of each denoised image.

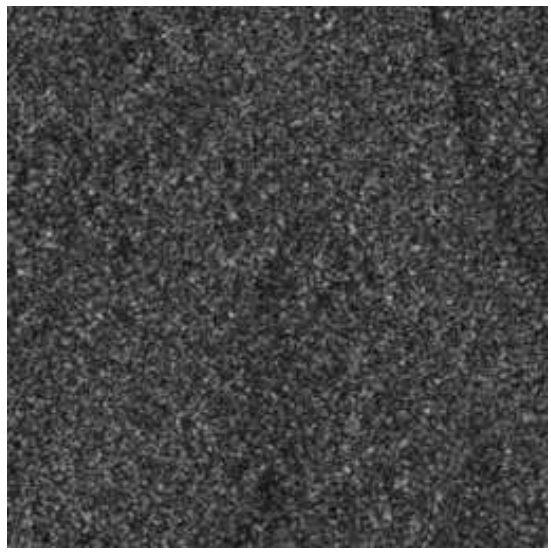

Initial 256x256 SAR image

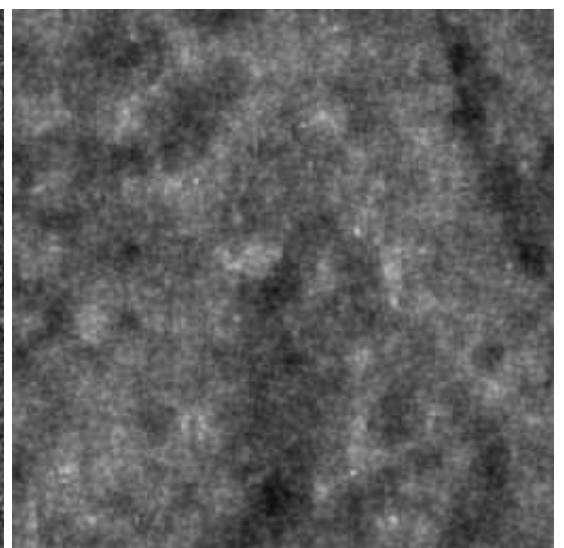

Denoised image using the multifractal IEA (the color dynamic has been adjusted).

Fig. 2. Results on a radar image in 14 generations. 


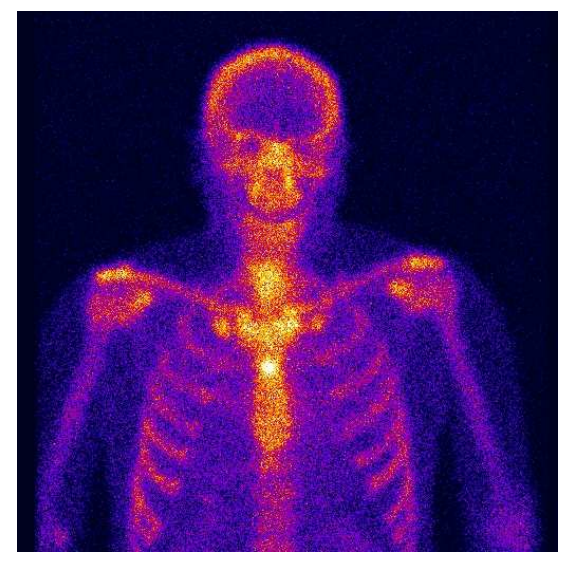

Initial noisy image

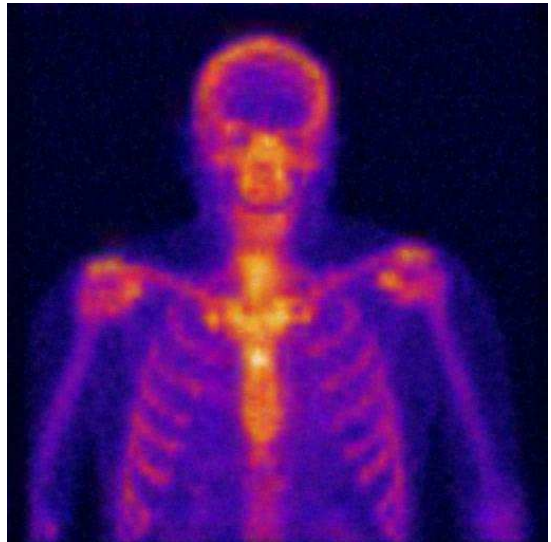

Multifractal IEA

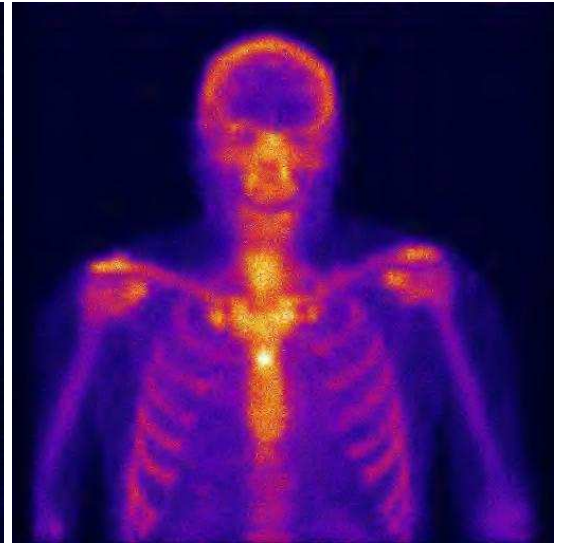

Soft thresholding

Fig. 3. Bones scintigraphy : 512x512 image

\section{Results}

Figure 2 shows a result of the interactive denoising on a radar image, figures 3 and 4 present comparative results with a soft thresholding technique.

Wavelet thresholding techniques consist in supressing the too small coefficients of the wavelet transform. There exist various thresholding procedures, the two most known ones are soft thresholding and hard thresholding. Hard thresholding consists in setting to 0 all the coefficients whose value are under a given threshold. Soft thresholding (also called wavelet shrinkage) lowers all the wavelet coefficient by a given quantity (the threshold), coefficients that are then negative are set to 0 . These techniques were proposed by D.Donoho and I.Johnstone in the beginning of 1990-ies [4]. 


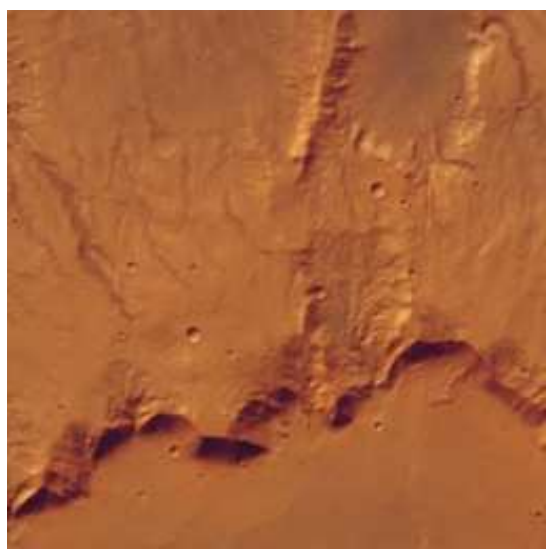

Original 256x256 image of Mars

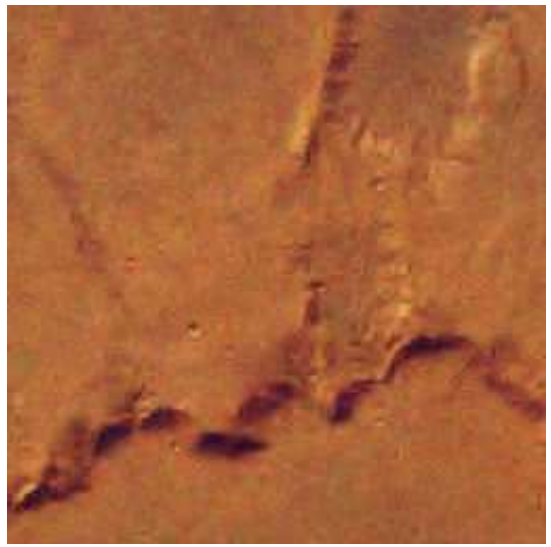

Multifractal IEA.

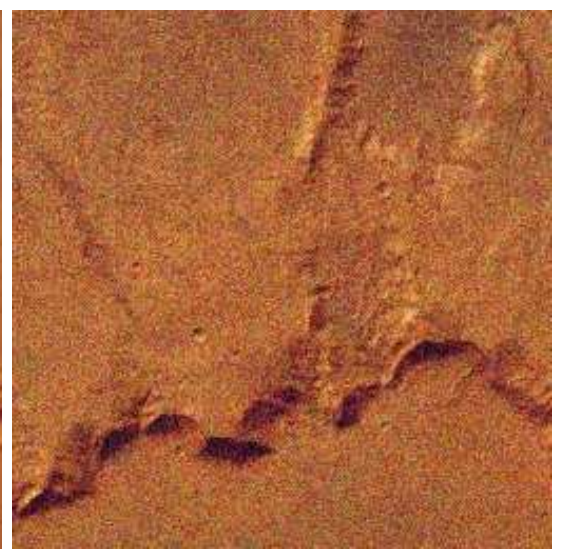

Noisy image (std dev 25)

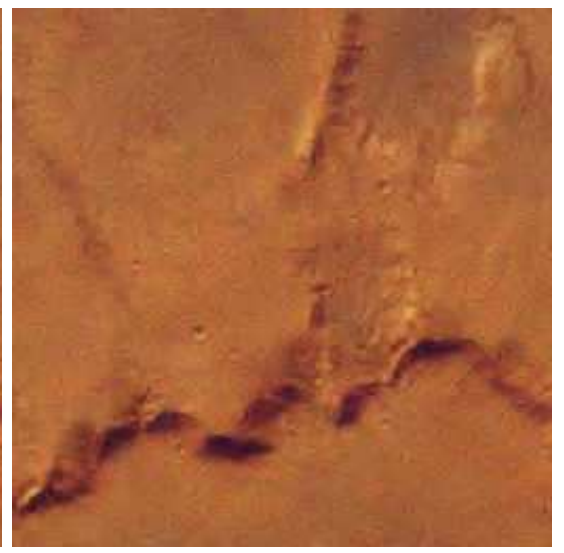

Soft thresholding.

Fig. 4. Comparative test with the interactive method

For fair comparison purpose, a non interactive version of the algorithm has been developed in order to test the multifractal denoising method: If the original noise-free image is available, an automatic fitness can be computed as the $L_{2}$ distance between denoised and original images. Successive populations of parameter setting can thus be evolved without user interaction. This process allows to obtain objective comparison data, even if the $L_{2}$ distance does not always reflect the visual impression of denoising quality.

Figure 5 shows an automatic experiment on the Lena image with a white Gaussian noise of variance 20 . The non-interactive EA has run during 300 generation. The initial distance between original and noisy image is 9119 . The distance obtained with the offline evolution after 300 generation was 4809 . A soft thresholding with optimal threshold yield a distance of 4935 , for a result that is visually very similar. 


\section{Conclusions and Future work}

Complex image processing techniques are often necessary for specialized purpose and/or "difficult" images. However their usage often necessitates a parameter tuning stage, that may be very combinatorial. The solution we propose is to assist the user in the search of its optimal parameter setting via IEA. Experiments have been produced here for a denoising application, that prove the versatility of the approach, and the efficiency of results in comparison to other denoising techniques.

We intend to continue in this direction for other multifractal image analysis methods. A version of the presented software will be soon availble in the fraclab toolbox (see http://fractales.inria.fr).

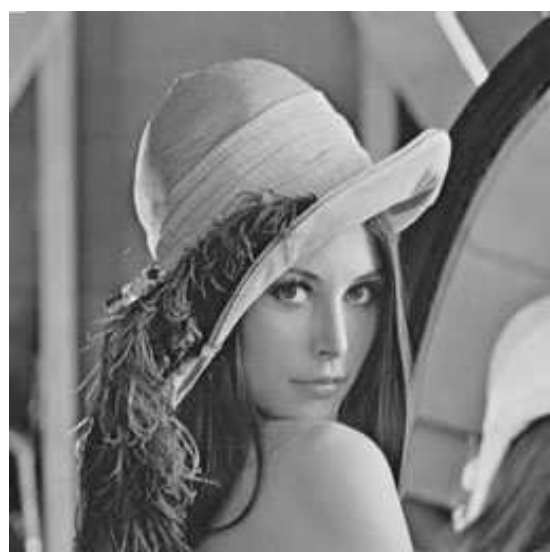

Original 256x256 image

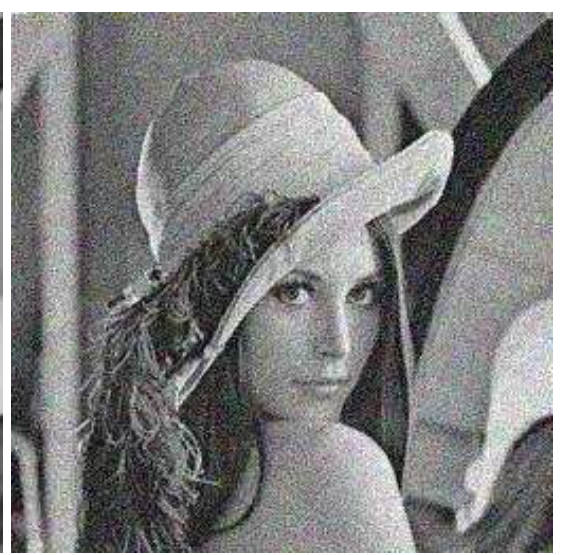

Noisy image (std dev 20)

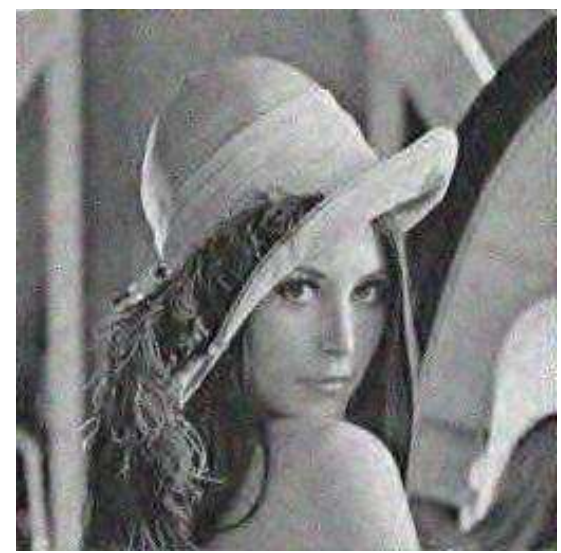

Denoised image using off-line evolution.

Fig. 5. Automatic tests on Lena 


\section{References}

1. P. J. Angeline. Evolving fractal movies. In Genetic Programming 1996: Proceedings of the First Annual Conference, John R. Koza and David E. Goldberg and David B. Fogel and Rick L. Riolo (Eds), pages 503-511, 1996.

2. Wolfgang Banzhaf. Handbook of Evolutionary Computation, chapter Interactive Evolution. Oxford University Press, 1997.

3. J. Chapuis and E. Lutton. Artie-fract : Interactive evolution of fractals. In 4th International Conference on Generative Art, Milano, Italy, December 12-14 2001.

4. D.L Donoho. De-noising by soft-thresholding. IEEE, Trans. on Inf. Theory, 3(41):613-627, 1995.

5. S. Kamohara, Hideyuki Takagi, and T. Takeda. Control rule acquisition for an arm wrestling robot. In IEEE Int. Conf. on System, Man and Cybernetics (SMC'97), volume 5, Orlando, FL, USA, 1997.

6. J. Lévy Véhel. Introduction to the multifractal analysis of images. In Y. Fisher, editor, Fractal Image Encoding and Analysis. Springer Verlag, 1997.

7. Jacques Lévy Véhel and Evelyne Lutton. Evolutionary signal enhancement based on hölder regularity analysis. In EVOIASP2001 Workshop, Como Lake, Italy, Springer Verlag, LNCS 2038, 2001.

8. Jacques Lévy Véhel and Pierrick Legrand. Bayesian multifractal signal denoising. In IEEE ICASSP Conference, 2003.

9. Nicolas Monmarche, G Nocent, Gilles Venturini, and P. Santini. Artificial Evolution, European Conference, AE 99, Dunkerque, France, November 1999, Selected papers, volume Lecture Notes in Computer Science 1829, chapter On Generating HTML Style Sheets with an Interactive Genetic Algorithm Based on Gene Frequencies. Springer Verlag, 1999.

10. Riccardo Poli and Stefano Cagnoni. Genetic programming with user-driven selection : Experiments on the evolution of algorithms for image enhancement. In 2nd Annual Conf. on Genetic Programming, 1997.

11. K. Sims. Interactive evolution of dynamical systems. In First European Conference on Artificial Life, pages 171-178, 1991. Paris, December.

12. Karl Sims. Artificial evolution for computer graphics. Computer Graphics, 25(4):319-328, July 1991.

13. Hideyuki Takagi. Interactive evolutionary computation : System optimisation based on human subjective evaluation. In IEEE Int. Conf. on Intelligent Engineering Systems (INES'98), Vienna, Austria, Sept 17-19 1998.

14. Hideyuki Takagi and Miho Ohsaki. Iec-based hearing aids fitting. In IEEE Int. Conf. on System, Man and Cybernetics (SMC'99), volume 3, Tokyo, Japan, Oct. 12-15 1999.

15. S.J.P. Todd and W. Latham. Evolutionary Art and Computers. Academic Press, 1992. 